Thorax (1968), 23, 210.

\title{
Spontaneous rupture of the oesophagus
}

\section{Endoscopic treatment in the primary stage}

\author{
R. A. H ARM $\ddot{A}$, Y. O. KOSKINEN, AND S. O. SUURKARI \\ From the Central Hospital, Kuopio, Finland
}

\begin{abstract}
A case of spontaneous rupture of the oesophagus occurring in the right wall of the midoesophagus is reported. The treatment, conservative in the primary stage of the disease, is described. This consisted of per-oesophageal drainage of the mediastinum, effected endoscopically. In spite of complications-an empyema in the right pleura and an oesophagocutaneous fistula-the treatment was successful, resulting in a complete cure about two years after the accident. The patient has remained in good health since then.
\end{abstract}

Spontaneous rupture of the oesophagus is a recognized entity which the Dutchman, Boerhave, was the first to describe in 1724. The clinical picture is of radiating pains in the chest or in the upper abdomen, beginning abruptly in connexion with some powerful exertion, usually heavy vomiting, which suddenly raises the intraabdominal pressure. The pain is followed by dyspnoea, cyanosis, and collapse. Cervical subcutaneous emphysema may also appear.

Increasingly better results have been obtained in the treatment of the disease since the introduction of thoracotomy and immediate repair of the rupture; this was first done successfully by Barrett in 1946 (Barrett, 1947). Without operative treatment the prognosis is bad, the mortality being $100 \%$ within one week. Tesler and Eisenberg (1963) reported that one-fourth of the cases died in the first 12 hours. By contrast, 60 to $70 \%$ of those treated by thoracotomy and primary suture recovered (Sealy, 1963; Tesler and Eisenberg, 1963). In our opinion, however, primary repair is not likely to succeed if operation is delayed for more than 12 hours after perforation: this is because the edges of the perforation are difficult to suture successfully. In cases where immediate thoracotomy has not been performed drainage of the pleural cavity or mediastinum with or without gastrostomy has been attempted, but with rather poor results (Ware, Shnider, and Davis, 1952).

In the past years only a few cases have been treated conservatively. Sanderson (1965) presented a patient treated with repeated thoracenteses and antibiotics. He stated that no well-documented case of survival after conservative treatment had been presented in the literature. Movsas (1966) reported a case of spontaneous cure following $\vec{\varphi}$ spontaneous oesophageal rupture. Recently, Hamilton (1967) has reported two cases treated successfully by conservative methods.

The purpose of this paper is to present a case of spontaneous rupture of the oesophagus which was treated successfully by an unorthodox method of therapy.

\section{CASE REPORT}

On 13 February 1963, P. I., a 35-year-old farmer, was admitted to the Central Hospital as an emergency. He was suffering chiefly from acute pain in the chest and upper abdomen.

HISTORY At the age of 2 years he had ingested a 0 small amount of lye but had not received any treat $-\underline{3}$. ment. Since then from time to time he had suffered from some dysphagia. Nevertheless, he had been able to eat all kinds of food.

About two hours before admission, after eating a 음 large meal of meat stew, he suddenly vomited $>$ violently. This was immediately followed by anㅡㅡㄹ. intense pain in the chest. Thinking he had a piece of food stuck in his gullet, the patient drank some? sour milk and wine as a remedy, but this made the $N$ symptoms worse.

PHYSICAL EXAMINATION On admission to hospital the patient was suffering from agonizing pain. He hade severe dyspnoea, and slight facial cyanosis was present. The blood pressure was high. Pulmonary? auscultation was normal, but auscultation of the $\square$ heart revealed a 'mediastinal crunch' (Hamman'sō sign). Remarkable rigidity and tenderness in the $\stackrel{\odot}{\circ}$

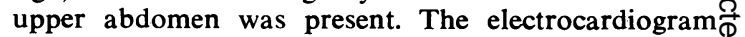
was normal. Radiological studies of the chest re- 0 vealed mediastinal emphysema (Fig. 1). During the examination a little fresh blood was vomited. 


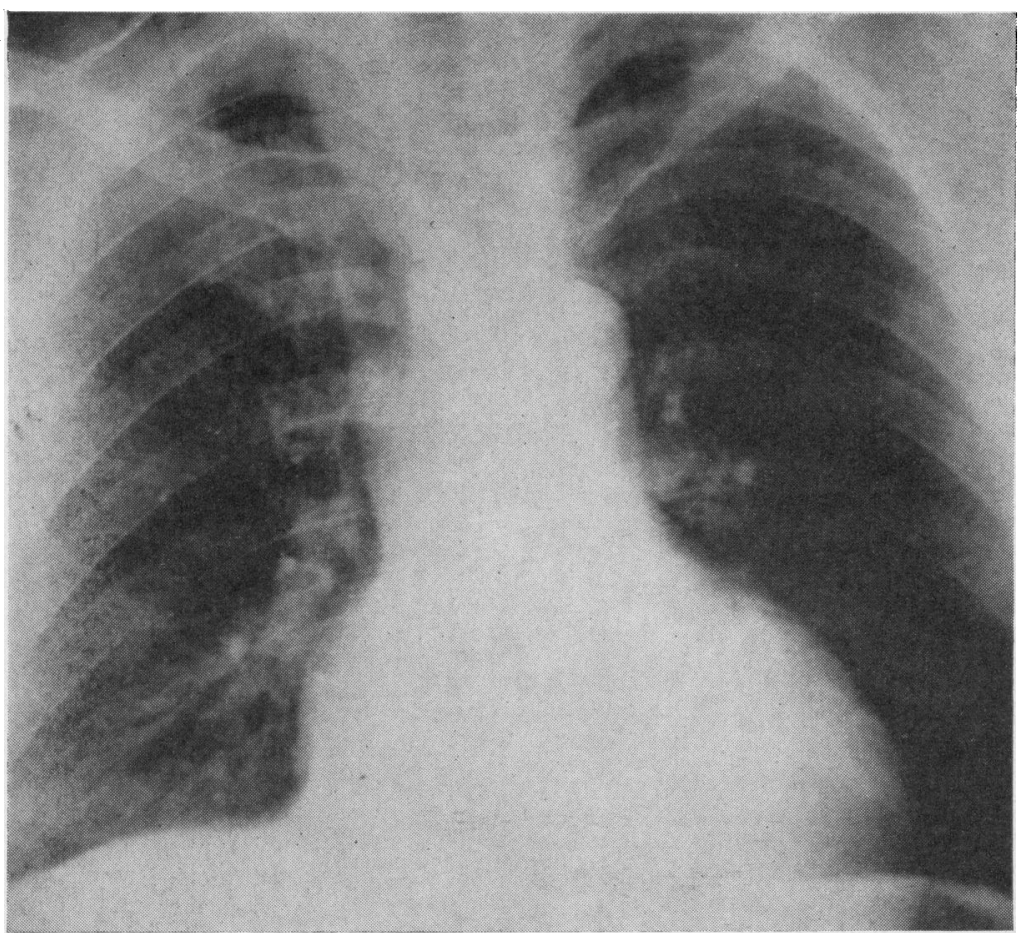

FIG. 1. Chest radiograph showing widening of the mediastinum and mediastinal emphysema on the right side.

An oesophageal rupture was suspected and an oesophagoscopy was performed. The oesophagoscopy revealed, in the middle portion of the oesophagus at a distance of $12 \mathrm{in}$. $(30 \mathrm{~cm}$.) from the upper teeth, a longitudinal perforation in the right wall. The tear measured 2 in. $(5 \mathrm{~cm}$.) in length. At oesophagoscopy a good deal of regurgitated gastric contents was removed from the mediastinum, through the perforation, by means of suction and forceps.

TREATMENT AND COURSE A correct diagnosis-spontaneous rupture of the oesophagus-was established after three hours had elapsed from the time of the accident, a time suitable for immediate surgical repair of the lesion. However, owing to unforeseen circumstances, no competent thoracic surgeon was available that night, which meant that treatment had to be conservative.

Treatment, which included intravenous administration of fluids and electrolytes as well as antibiotics, was immediately started. Another oesophagoscopy was performed a few hours after the first. In addition to the rupture, a mild stricture was found in the lower portion of the oesophagus just above the hiatus; the stricture was easily dilated with bougies up to no. 29 . A $\frac{5}{16}$ in. $(7.9 \mathrm{~mm}$.) naso-gastric feeding tube was inserted into the oesophagus under direct visual control. Likewise, another tube was inserted into the mediastinum through the perforation. Both these tubes were brought out through the nose and secured. Both tubes were also tied together in the pharynx in order to keep the mediastinal tube fixed. The mediastinal tube was kept closed, except for the instillation of antibiotics into the mediastinal cavity; and intermittent suction was applied to this tube at 30-minute intervals. For the first two days the secretion from the mediastinal tube was slight, but from the third day on the secretion became abundant and pus-like.

Repeated radiological studies of the chest were made, and after one week a left hydropneumothorax was present. On 20 February a thoracentesis was made and permanent underwater seal drainage was applied to the right pleural cavity. On 2 March the chest radiograph revealed that the empyema had resolved and the drainage tube was removed. After a couple of days, however, the right lung was found to have collapsed. Therefore on 8 March a minor thoracotomy was performed by resecting a small portion of the eighth rib. At operation some remnants of food were removed from the pleural cavity. The pleura had thickened, and the lung did not expand to its former size even with overpressure. The lower end of the tube inserted through the rupture into the 


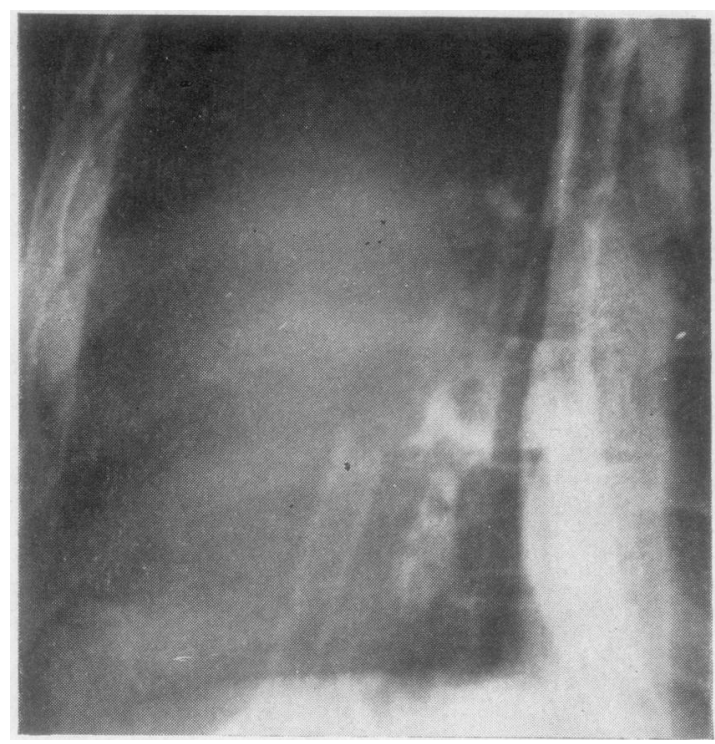

FIG. 2. Propyliodone swallow showing a fistula from the middle of the oesophagus to the right pleura.

mediastinum was seen in the pleural space. This tube was removed and drainage was continued by closing the chest with underwater seal drainage.

On 15 March a contrast study revealed an oesophagopleurocutaneous fistula (Fig. 2). Ten days later an oesophagoscopy was performed. It revealed at the site of the former perforation a diverticulum-like funnel-shaped pouch that seemed to have a blind end and a depth of about 0.5 in. (12 mm.). Next day this was confirmed by a contrast medium swallow, in which the funnel-shaped pouch was visible (Fig. 3). The naso-gastric tube was removed and the patient began to take his food orally six weeks after his illness had begun.

The secretion from the pleural cavity diminished little by little until no secretion was obtained by suction, and on 10 April the pleural drain was removed. Next day the patient was discharged from hospital in good condition, eight weeks after admission. The empyema, however, soon reappeared and only healed finally after two years, when a minor thoracoplasty was performed by resecting a portion of the seventh and eighth ribs on 4 March 1965. Since then the patient has been in good health without any complaints.

The patient's general condition began to improve as soon as treatment was begun. After a couple of days he felt no pain even without analgesics. On the second day in hospital he was able to take short walks in his room. The febrile period (the temperature ranging to $102^{\circ} \mathrm{F}$.) lasted for four days and the temperature remained subfebrile for a total of nine days. The administration of sterile liquids through the nasogastric tube was started on the second day, and after four days all the food was given by this method.
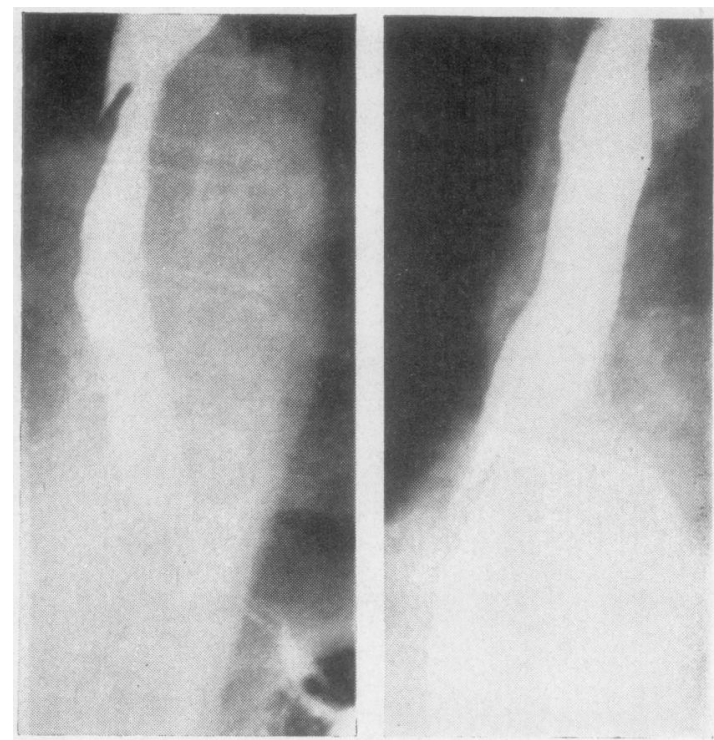

FIG. 3. Barium swallow 10 days later. A small pouch is seen at the site of the former fistula.

\section{DISCUSSION}

We believe that spontaneous rupture of the oesophagus can occur without pre-existing oesophageal disease ; but in several instances there has been some underlying condition favouring the occurrence of the rupture (Meagher, Lupien, and Albert, 1962). Thus, the presence of a mild stricture at the lower end of the oesophagus in our patient does not prevent the authors from using the term 'spontaneous'. A precipitating factor for rupture is increased intra-abdominal pressure, caused by vomiting in $85 \%$ of cases (Ware et al. 1952).

We should like to discard the term 'spontaneous' and to use instead 'pressure rupture'. We would retain the term 'spontaneous' for such cases as true perforation of peptic ulcer of the oesophagus, as suggested by Clark and Tankel (1964). This distinction between the two types of lesion was also made by Mackler (1952).

The site of the rupture in our patient in the right wall is uncommon; over $95 \%$ of ruptures occur in the lower third of the oesophagus and on the left postero-lateral aspect (Bowers and Ferguson, 1966). Tidman and John (1967) recently reported seven cases of spontaneous rupture of the oesophagus, five of these occurring in the right wall. According to them, only four cases of rightsided rupture of the mid-gullet had been reported before their series. 
The treatment in our case was unusual. We have not been able to find any report of oesophageal rupture treated by per-oesophageal drainage of the mediastinum. The primary stage, the usually fatal first week in cases treated nonoperatively (Derbes and Mitchell, 1956 ; Kinsella, Morse, and Hertzog, 1948 ; Ware et al., 1952), was controlled very well by this method. After one week an empyema appeared in the right pleura, and thoracentesis with the application of permanent suction was needed. The oesophagopleurocutaneous fistula that appeared after the removal of the per-oesophageal drainage tube was a deficiency of pleural drainage. The fistula that developed closed quickly-in fact, only two weeks after removal of the tube the fistula was shown to be completely closed. Oesophagocutaneous fistula is a common eomplication in cases treated by primary operation (Derbes and Mitchell, 1956 ; Hardin, Hardy, and Conn, 1967 ; Tesler and Eisenberg, 1963), as is post-operative empyema (Sealy, 1963 ; Tesler and Eisenberg, 1963).

The authors recognize the superiority of immediate surgical intervention in the treatment of spontaneous oesophageal rupture; but in patients in whom this is out of the question the method described above can succeed.

We endorse the opinion of Kyser (1964) 'It is the mature clinician who recognizes the condition promptly after onset and institutes immediate and life-saving therapy', even if the therapy is only the best that one can offer, and not perhaps the best conceivable.

\section{REFERENCES}

Barrett, N. R. (1947). Report of a case of spontaneous perforation of the oesophagus successfully treated by operation. Brit. J. Surg., 35, 216.

Bowers, C. R., and Ferguson, D. H. (1966). Rupture of the esophagus Amer. J. Surg., 111, 175.

Clark, D. H., and Tankel, H. I. (1964). Pressure rupture and spontaneous perforation of the oesophagus. Gut, 5,86 .

Derbes, V. J., and Mitchell, R. E. (1956). Rupture of the esophagus. Surgery, 39, 688.

Hamilton, S. G. I. (1967). Spontaneous rupture of the oesophagus. Brit. J. Surg., 54, 304.

Hardin, W. J., Hardy, J. D., and Conn, J. H. (1967). Esophagea I perforations. Surg. Gynec. Obstet., 124, 325.

Kinsella, T. J., Morse, R. W., and Hertzog, A. J. (1948). Spontaneous rupture of the esophagus. J. thorac. Surg., 17, 613 (quoted by Sealy, 1963).

Kyser, F. A. (1964). Spontaneous perforation of the esophagus. Med. Clin. N. Amer., 48, 3.

Mackler, S. A. (1952). Spontaneous rupture of the esophagus. Surg. Gynec. Obstet., $95,345$.

Meagher, R. P., Lupien, J., and Albert, S. N. (1962). Postoperative rupture of the esophagus. Ibid., 115, 677.

Movsas, S. (1966). Spontaneous rupture of the oesophagus: Is conservative treatment ever justified? Thorax, 21, 111.

Sanderson, R. G. (1965). Spontaneous rupture of the esophagus. Amer. J. Surg., 109, 506.

Sealy, W. C. (1963). Rupture of the esophagus. Ibid., 105, 505.

Tesler, M. A., and Eisenberg, M. M. (1963). Spontaneous esophageal rupture. Surg. Gynec. Obstet., 117, Int. Abstr. Surg., 1.

Tidman, M. K., and John, H. T. (1967). Spontaneous rupture of the oesophagus. Brit. J. Surg., 54, 286.

Ware, G. W., Shnider, B. I., and Davis, E. W. (1952). Spontaneous rupture of the esophagus. Arch. Surg., 65, 723. 